\title{
RESEARCH
}

Open Access

\section{A1 astrocytes contribute to murine depression-like behavior and cognitive dysfunction, which can be alleviated by IL- 10 or fluorocitrate treatment}

He-Yang Zhang ${ }^{1 \dagger}$, Yan Wang ${ }^{1 \dagger}$, Youdi He ${ }^{3 \dagger}$, Ting Wang ${ }^{1}$, Xiao-Hui Huang ${ }^{1}$, Chang-Ming Zhao ${ }^{1}$, Lei Zhang ${ }^{4}$, Si-Wei Li ${ }^{1}$, Changyong Wang ${ }^{1}$, Yan-Nv Qu ${ }^{1,5^{*}}$ and Xiao-Xia Jiang ${ }^{1,2^{*}}$

\begin{abstract}
Background: Astrocytes are crucial regulators in the central nervous system. Abnormal activation of astrocytes contributes to some behavior deficits. However, mechanisms underlying the effects remain unclear. Here, we studied the activation of A1 astrocytes and their contribution to murine behavior deficits.
\end{abstract}

Methods: A1 astrocytes were induced by treatment with lipopolysaccharide (LPS) in vitro. The functional phenotype of astrocytes was determined by quantitative RT-PCR, ELISA, and immunohistochemistry. To assess the role of A1 astrocytes in vivo, mice were injected intraperitoneally with LPS. Then, murine behaviors were tested, and the hippocampus and cortex were analyzed by quantitative RT-PCR, ELISA, and immunohistochemistry. The function of IL-10 and fluorocitrate on A1 astrocyte activation was also examined.

Results: Our results show that astrocytes isolated from B6.129S6- $\| 10^{\mathrm{tm} 1 \mathrm{Flv}} / \mathrm{J}$ homozygotes $\left(\mathrm{IL}-10^{\mathrm{tm} 1 / \mathrm{tm} 1}\right)$ were prone to characteristics of $\mathrm{A} 1$ reactive astrocytes. Compared with their wild-type counterparts, $\mathrm{IL}-10^{\mathrm{tm} 1 / \mathrm{tm} 1}$ astrocytes exhibited higher expression of glial fibrillary acidic protein (GFAP). Whether or not they were stimulated with LPS, $\mathrm{IL}-10^{\mathrm{tm} 1 / \mathrm{tm} 1}$ astrocytes exhibited enhanced expression of A1-specific transcripts and proinflammatory factors IL-1 $\beta$, $\mathrm{IL}-6$, and TNFa. In addition, IL-10 $0^{\mathrm{tm} / \mathrm{tm} 1}$ astrocytes demonstrated hyperphosphorylation of STAT3. Moreover, astrocytes from $\mathrm{IL}-10^{\mathrm{tm} 1 / \mathrm{tm} 1}$ mice showed attenuated phagocytic ability and were neurotoxic. $\mathrm{IL}-10^{\mathrm{tm} 1 / \mathrm{tm} 1}$ mice demonstrated increased immobility time in the forced swim test and defective learning and memory behavior in the Morris water maze test. Moreover, enhanced neuroinflammation was found in the hippocampus and cortex of $\mathrm{IL}-10^{\mathrm{tm} 1 / \mathrm{tm} 1}$ mice, accompanying with more GFAP-positive astrocytes and severe neuron loss in the hippocampus. Pretreatment IL-10 ${ }^{\mathrm{tm} 1 / \mathrm{tm} 1}$ mice with $\mathrm{IL}-10$ or fluorocitrate decreased the expression of proinflammatory factors and A1-specific transcripts in the hippocampus and cortex, and then alleviated LPS-induced depressive-like behavior.

(Continued on next page)

\footnotetext{
* Correspondence: nacyququ@163.com; smilovjiang@163.com

${ }^{+} \mathrm{He}$-Yang Zhang, Yan Wang and Youdi He contributed equally to this work. 'Department of Neural Engineering and Biological Interdisciplinary Studies, Institute of Military Cognition and Brain Sciences, Academy of Military Medical Sciences, 27 Taiping Road, Haidian District, Beijing 100850, China Full list of author information is available at the end of the article
} 
(Continued from previous page)

Conclusion: These results demonstrate that astrocytes isolated from B6.129S6- $\| 10^{\mathrm{tm} 1 \mathrm{Flv}} / \mathrm{J}$ homozygotes are prone to

A1 phenotype and contribute to the depression-like behavior and memory deficits. Inhibiting A1 astrocyte

activation may be an attractive therapeutic strategy in some neurodegenerative diseases.

Keywords: A1 astrocytes, IL-10, Neuroinflammation, Behavior deficits, Cognitive dysfunction

\section{Introduction}

Astrocytes comprise the most abundant glial cells in the mammalian brain. They are critical regulators of brain development and perform many functions important for normal neuronal survival and growth, synapse formation, and proper propagation of action potentials [1-3].

There is increasing evidence that astrocytes in different brain regions are not identical, and even neighboring astrocytes within the same brain region may have differences [4-6]. Reactive astrocytes, a common pathological response during the repair and scarring of the brain following injury, are often associated with brain metastases in animal models and human patients [7]. Reactive astrocytes have been shown to enhance tumor cell proliferation, survival, invasive capabilities, and resistance to chemotherapy [7]. Recent studies have shown that neuroinflammation and ischemia induced two different types of reactive astrocytes, termed "A1" and "A2" [810]. A1 neuroinflammatory reactive astrocytes have been previously shown to be destructive to synapses, suggesting that A1 might have "harmful" functions. By contrast, ischemia-induced A2 reactive astrocytes upregulated many neurotrophic factors, which promote survival and growth of neurons, as well as thrombospondins, which promote synapse repair. This upregulation suggests that A2 might have trophic reparative functions. The finding that different types of reactive astrocytes are induced by different types of injury-and that ischemic injury produces a so-called helpful "A2" reactive astrocyte, whereas an inflammatory insult produces a more harmful "A1" reactive astrocyte-raises many questions [10-12]. A more comprehensive understanding of the heterogeneity of reactive astrocytes still requires a more thorough investigation.

IL-10 is a key cytokine that represses excessive inflammatory responses links with anti-inflammatory and protective functions in the central nervous system (CNS) [13]. In the CNS, IL-10 is mainly produced by astrocytes and microglia and it is upregulated after various insults. Astrocyte-targeted production of IL-10 attenuated the microglial response and culminated in a beneficial effect on neuronal survival [14-16]. Adult neural stem cells (NSCs) engineered to express IL-10 exhibited enhanced capacity to suppress immune response, promote remyelination, and neuronal repair [17]. In addition to ameliorate chronic CNS inflammation, IL-10 modulates progenitor differentiation and regulates neurogenesis $[18,19]$. However, elevated IL-10 is not always beneficial. In some Alzheimer's disease (AD) patient brains, IL-10 signaling pathway was abnormally increased. In APP/PS1 mice, IL-10 deficiency was shown to partially rescue synaptic toxicity and mitigate behavioral impairment [20]. IL-10 overexpression in the brains of APP transgenic mice resulted in increased $A \beta$ accumulation and impaired memory [21]. The complex effect of IL-10 on innate immune activation status in the brain and proteostasis and neurodegenerative diseases indicates controlling the right dose of IL-10 under right condition is critical for brain development and function.

In the present study, we found astrocytes from B6.129S6-Il10 ${ }^{\mathrm{tm} 1 \mathrm{Flv}} / \mathrm{J}$ homozygotes (IL-10 ${ }^{\mathrm{tm} 1 / \mathrm{tm} 1}$ ), which have lower IL-10 expression, were prone to A1 astrocytes, showing as higher levels of A1 transcripts H2-T23, H2-D1, Gbp2, and pro-inflammatory factors, attenuated normal astrocytic protective functions. After treated with lipopolysaccharide (LPS), IL-10 ${ }^{\mathrm{tm} 1 / \mathrm{tm} 1}$ homozygotes showed enhanced neuroinflammation; elevated expression of A1 transcripts H2-T23, H2-D1, and Gbp2; and worse behavioral deficits. Exogenous IL-10 or fluorocitrate (FC) inhibited A1 astrocyte activated and ameliorated LPS-induced behavior deficits.

\section{Material and methods \\ Animals}

Groups of 8-12-week-old C57BL/6 mice were obtained from the Laboratory Animal Center of the Academy of Military Medical Sciences of China (Beijing). B6.129S6Il10 ${ }^{\mathrm{tm} 1 \mathrm{Flv} / \mathrm{J}}$ mice [22] were from Prof. Jianli Wang at Zhejiang University. In all experiments, age- and sexmatched wild-type (WT) littermates were used for controls. Mice were maintained in a pathogen-free barrier facility. All animal experiments were performed according to the Guide for the Care and Use of Laboratory Animals and were approved by the Institute of Military Cognition and Brain Sciences. The institutional Ethics Review Committee for Animal Experimentation approved all experimental protocols.

\section{Primary cell culture}

Primary astrocytes were prepared from newborn C57BL/ 6 or B6.129S6-Il10 ${ }^{\mathrm{tm} 1 \mathrm{Flv} / \mathrm{J}}$ pups. After the removal of 
meninges, the cortical tissues were mechanically minced and dissociated with $0.25 \%$ trypsin (Gibco). After trypsin inactivation, the tissue suspension was filtered through a 70-mm nylon cell strainer. Cell pellets were harvested and resuspended in Dulbecco's modified Eagle medium/ nutrient mixture F-12 (DMEM/F12) (1:1) (Gibco) supplemented with $10 \%$ heat-inactivated FBS, $100 \mathrm{U} / \mathrm{ml}$ penicillin, and $100 \mu \mathrm{g} / \mathrm{ml}$ streptomycin and plated on poly-L-lysine pre-coated culture flasks. Following incubation overnight in a humidified incubator at $37^{\circ} \mathrm{C}$ and $5 \% \mathrm{CO}_{2}$, the flasks were vigorously shaken to detach neurons and microglia. Culture medium was replaced every 3 days thereafter until the cell monolayer reached confluence. Primary neurons were obtained by microdissection from brains of neonatal C57BL/6 pups using the same protocol but cultured in neurobasal medium supplemented with 1\% glutamate and 2\% B27 (Gibco). Primary microglial cells were also dissected from neonatal C57BL/6 pups. After removing the meninges, brains were mechanically minced and dissociated with $0.25 \%$ trypsin. The tissue suspension was passed through a 70$\mathrm{mm}$ nylon cell strainer. Cell pellets were harvested and resuspended in DMEM supplemented with 10\% heatinactivated FBS and plated on poly-L-lysine precoated culture flasks. Primary microglial cells were harvested by shaking (200 rpm, $20 \mathrm{~min}$ ) after overnight in culture.

\section{Quantitative PCR}

Cells were collected in TRIzol (Sigma-Aldrich), and total RNA were prepared by chloroform extraction and isopropanol precipitation according to the manufacturer's recommendations (Invitrogen). cDNA was used as a template in quantitative PCR with SYBR Green (Toyobo) to determine specific gene expression. Real-time RTPCR was performed using a SYBR Green PCR mix and conducted with the FTC-3000. Primer sequences were as follows: Aldh1l1 (forward, AGCAGAGGCCATTC ACAACT; reverse, GCCACCAGTCCTGAAGTGTT), Aqp4 (forward, CTCCCTTTGCTTTGGACTCA; reverse, CGATGCTGATCTTTCGTGTG; GFAP: forward, GCCACCAGTAACATGCAAGA; reverse, GGCGAT AGTCGTTAGCTTCG), Vim (forward, CTGCACGATGAAGAGATCCA; reverse, AGCCACGCTTTCAT ACTGCT), Aif1 (forward, CCGAGGAGACGTTCAGCT AC; reverse, GACCAGTTGGCCTCTTGTGT). Cx3cr1 (forward, AGCTCACGACTGCCTTCTTC; reverse, GT CCGGTTGTTCATGGAGTT), Snap25 (forward, GAG CAGGTGAGCGGCATCATC; reverse, CGTTGGTTGG CTTCATCAATTCTGG; Syt1: forward, GACTAT GACAAGATTGGCAAGAACGAC; reverse, ATGGCA TCAACCTCCTCCTCTACC), H2-T23 (forward, TG ATCATCCTTGGAGCTGTG; reverse, TTCTGAGGCC AGTCAGAGGT), Serping1 (forward, GCTCTACCAC GCCTTCTCAG; reverse, GGATGCTCTCCAAGTTGC
TC). H2-D1 (forward, GTTGCTGTTCTGGGTGTCCT; reverse, CCTGGAGCCAGAGCATAGTC), Gbp2 (forward, GGAGGAGCTGTGTGGTGAAT; reverse, TTAGACGTGGCCCATTGACT; Tgm1: forward, CCCTGG ATGACAATGGAGTT; reverse, GAATAGCCGGTGCG TAGGTA), Ptx3 (forward, TTCCTGAGGGTGGA CTCCTA; reverse, CCGATCCCAGATATTGAAGC), S100a10 (forward, GTGCTCATGGAACGGGAGT; reverse, AAAGCTCTGGAAGCCCACTT). Gpc4 (forward, GGTGACTGTGAAGCCCTGTT; reverse, TCTCTG CCACCATCAGCATA), Gpc6 (forward, AGCATT GCCCTACACCATCT; reverse, AGCCCATCGTTCAT GATCTC; Sparcl1: forward, AAACCATCCCAGTG ACAAGG; reverse, TCCTCATCCTTCAGGTCCAC), Thbs1 (forward, TGATGACTACGCTGGCTTTG; reverse, TGAGTATCCCTGAGCCCTTG), Thbs2 (forward, CTGGTGCAGACAGCAAACTC; reverse, CA AAGCCAGCGTAGTCATCA). Megf10 (forward, CTGGCGCTGTTCATCATCTA; reverse, CAGGGT TTCTGCGATGGTAT), Mertk (forward, CACGAG AACAACCTTCGTGA; reverse, TCCTCCGGTAAGAC ACCATC; Gas6: forward, AGGCCACCCTAGAAGTGG AT; reverse, CAGGCCTCCAACATAGGTGT), Axl (forward, GGAAGGTCAGCTCAATCAGG; reverse, CC TTCATGCAGACAGCTTCA), IL-1 $\beta$ (forward, GT GGCTGTGGAGAAGCTGTGG; reverse, CGGAGC CTGTAGTGCAGTTGTC), IFN $\gamma$ (forward, CAGGCC ATCAGCAACAACATAAGC; reverse, AGCTGGTGGA CCACTCGGATG; IL-6: forward, ACTTCCATCCAGTT GCCTTCTTGG; reverse, TTAAGCCTCCGACTTGTG AAGTGG), TNF $\alpha$ (forward, GATGGGTTGTACCTTG TCTACT; reverse, CTTTCTCCTGGTATGAGATA GC), iNOS (forward, CAGCTGGGCTGTACAAACCTT; reverse, CATTGGAAGTGAAGCGTTTCG), GAPDH (forward, TCACCATCTTCCAGGAGCGAGAC; reverse, AGACACCAGTAGACTCCACGACATAC).

\section{Immunofluorescence}

Brain processing and immunostaining were performed on free-floating sections. Mice were anesthetized with 2,2,2tribromoethanol (350 mg/kg, Sigma-Aldrich, T48402) and transcardially perfused with $0.9 \%$ saline, and brains were removed and fixed in phosphate-buffered $4 \%$ paraformaldehyde for $48 \mathrm{~h}$ before cryoprotection with $30 \%$ sucrose. Mouse brain sections $(30 \mu \mathrm{m})$ or fixed cultured cells were washed three times with PBS, and antigen retrieval was performed using citrate buffer ( $\mathrm{pH}$ 7.0); samples were then permeabilized and blocked in PBS containing 0.5\% Triton $\mathrm{X}-100$ and $10 \%$ normal goat serum at room temperature for $1 \mathrm{~h}$. Sections were incubated with primary antibodies in blocking buffer overnight at $4{ }^{\circ} \mathrm{C}$. After washing, secondary antibodies were added to the blocking buffer and incubated for $1 \mathrm{~h}$. Samples were then washed and counterstained with 4',6-diamidino-2-phenylindole (DAPI). 
Images were acquired under a fluorescence microscope (Nikon AZ-100 multipurpose microscope). Primary antibodies used for immunostaining include GFAP (mouse, 1: 500; Abcam, ab10062), IBA1 (Rabbit, 1:300; Abcam, ab178847), and MAP2 (goat, 1:500; Abcam, ab32454). Donkey anti-mouse/rabbit 488/594 secondary antibodies (1:1000) and mounting medium with DAPI were purchased from Invitrogen.

\section{Western blot}

Cells were lysed with lysis buffer, and protein samples were separated on $12 \%$ SDS-polyacrylamide gel, and then the proteins were transferred to $0.45 \mu \mathrm{m}$ polyvinylidene fluoride blotting membranes. The membrane was blocked in 5\% non-fat dry milk for $1 \mathrm{~h}$ and was then probed overnight at $4{ }^{\circ} \mathrm{C}$ with mouse anti-GFAP $(1: 1000$, Abcam, ab10062), rabbit anti-p-STAT3 (1:1000, Cell Signaling Technology, \#9167), mouse anti-STAT3 (1:1000, Cell Signaling Technology, \#9139), or rabbit antiGAPDH (1:1000, Cell Signaling Technology, \#1228). Blots were incubated with HRP-conjugated secondary antibodies at 1:5000 for $2 \mathrm{~h}$ at room temperature and developed using enhanced chemiluminescence substrate (Thermo Fisher, Waltham, MA, USA). Visualization and imaging of blots was performed with a FluorochemQ System (ProteinSimple).

\section{Neuron survival assays}

Astrocytes were treated with or without LPS $(100 \mathrm{ng} / \mathrm{mL}$, L-4391, Sigma-Aldrich) for $24 \mathrm{~h}$, and then, supernatants were collected. Neurons were plated at 150,000 cells per well on PDL-coated plastic coverslips in 24-well cell culture plates. Supernatants were added to neurons and viability assessed at indicated time point.

\section{$A \beta$ phagocytosis}

For phagocytosis assays, astrocytes pretreated with or without LPS $(100 \mathrm{ng} / \mathrm{mL}$ for $24 \mathrm{~h})$ were incubated with aggregated $400 \mathrm{nM}$ Hilyte555-A 342 (Anaspec, Fermont, USA). Cells were analyzed at indicated time points following addition of $A \beta$ to the culture.

\section{LPS administration}

Lipopolysaccharide (LPS, L-4391, Sigma-Aldrich) was dissolved in physiological saline. The dose of LPS $(0.83$ $\mathrm{mg} / \mathrm{kg}$ ) and the time point $(24 \mathrm{~h})$ for behavioral tests after LPS intraperitoneal injection (i.p.) were selected based on previous investigations [23].

\section{Hippocampus and cortex protein extraction}

Snap-frozen hippocampus and cortex were homogenized in tissue protein extraction reagent (T-PER, Thermo Fisher Scientific) containing a mixture of protease and phosphatase inhibitors (Thermo Fisher Scientific).
Homogenates were centrifuged, and the supernatants were evaluated for the quantification of cytokines.

\section{ELISA quantification of cytokines}

Cytokines were quantified with custom Mouse ProcartaPlex ${ }^{\mathrm{Tu}}$ Panel according to the manufacturer's instructions (eBioscience). Data acquisition was performed with a MAGPIX (Luminex). Each sample was measured in duplicate.

\section{IL-10 and fluorocitrate injection}

Mice were anesthetized with 2,2,2-Tribromoethanol $(350 \mathrm{mg} / \mathrm{kg}$, Sigma-Aldrich, T48402) intraperitoneal injection, then placed into a stereotaxic frame. A small Hamilton syringe (33-gauge) was used to slowly inject $1 \mu \mathrm{l}$ of saline or IL-10 (20 ng, Peprotech) or fluorocitrate (FC, $1 \mathrm{nmol}$, Sigma-Aldrich) per mouse into intracerebroventricular (i.c.v). After injection, the syringe was held in place for $5 \mathrm{~min}$ to avoid back-flow of cerebrospinal fluid (CSF). The stereotaxic coordinates were 1.34 $\mathrm{mm}$ posterior, $0 \mathrm{~mm}$ lateral to the bregma, and $2.3 \mathrm{~mm}$ ventral to the bregma.

\section{Forced swim test (FST)}

Mice were individually placed in a glass cylinder $(25 \mathrm{~cm}$ height, $15 \mathrm{~cm}$ diameter) filled with room temperature water $\left(23^{\circ} \mathrm{C}-25^{\circ} \mathrm{C}\right)$. Mice were allowed to swim inside the cylinder and videotaped for $6 \mathrm{~min}$. The duration of immobility in the last 4 min was counted by an observer blinded to the animal treatments. Immobility was defined as time when animals remained floating or motionless with only movements necessary for keeping balance in the water.

\section{Morris water maze test (MWM)}

The water maze used in this study comprised a circular tank $120 \mathrm{~cm}$ in diameter with a platform filled with tap water at a temperature of $22 \pm 2{ }^{\circ} \mathrm{C}$. Reference cues with different colors and shapes were posted along the walls surrounding the tank. Within the tank was a fixed platform (diameter, $10 \mathrm{~cm}$ ) located in a target quadrant. A camera was mounted above the maze to record the swimming traces in the water maze. During testing, the platform was submerged, and 1-2 $\mathrm{cm}$ below the water surface, mice were placed into the maze at one of four points $(\mathrm{N}, \mathrm{S}, \mathrm{E}, \mathrm{W}) \mathrm{fa}$ cing the wall of the tank. Mice were provided $60 \mathrm{~s}$ to search for the platform. If a mouse failed to find the platform, it was guided to the platform and maintained on the platform for $10 \mathrm{~s}$. Four trials per day were conducted with an intermission of $1 \mathrm{~h}$ minimum between trials. Between the trails, mice were gently padded dry and warmed on a heating pad. The escape latency, or the time it took for the mouse to reach the platform, was scored for each trial and averaged per testing day. On day 6, the platform was removed and a probe test was performed. The percentage of time spent in each of the four quadrants and the number of 
platform area crossings, mean speed, and total distance were recorded.

\section{Statistical analysis}

All data were analyzed with Prism 5.0 software (GraphPad Software, San Diego, CA, USA) and are presented as means \pm standard deviations (SDs). Statistical significance was assessed by unpaired two-tailed Student's $t$ tests. For multiple comparisons, two-way ANOVA was performed, followed by Bonferroni multiple comparison. $\left({ }^{*} p<0.05 ;{ }^{* *} p<0.01\right)$.

\section{Results}

A1-specific transcripts were induced in astrocytes with inflammatory factors

Previous studies have shown that A1 astrocytes can be induced by multiple inflammatory factors [8]. To confirm the activation state of astrocytes with different stimulators, we first isolated astrocytes from neonatal mice and used quantitative RT-PCR to determine lineage-specific gene expression. As expected, astrocyte-specific markers Aldh1l1, glial fibrillary acid protein (GFAP), Vim, and Aqp4 were highly expressed, while microglia marker Cx3cr1, Aif1 and neuron marker Snap25, Syt1 were almost undetectable (Fig. 1a, top). GFAP and microglial marker, ionized calcium-binding adaptor molecule 1 (IBA1) revealed that no microglial cells were detected in astrocytic cultures, excluding a potential contribution of microglia in the culture system (Fig. 1a, bottom). Astrocytes can be activated by various stimulators. We treated the isolated astrocytes with different doses of LPS, TNF $\alpha$, IL- $1 \alpha$ plus TNF $\alpha$, TGF- $\beta 1$, FGF, and IL- $1 \beta$, respectively, for $24 \mathrm{~h}$. As shown in Fig. 1b, A1-specific transcripts H2-T23, H2-D1, and Gbp2 were significantly increased under treatment with LPS, TNF $\alpha$, IL-1 $\alpha$ plus TNF $\alpha$, and IL-1 $\beta$. However, A2-specific transcripts only increased significantly under IL-1 $\beta$ treatment. Since LPS at $100 \mathrm{ng} / \mathrm{ml}$ can induce high expression of A1-specific transcripts, the stimulation condition was chosen for the following studies.

\section{Astrocytes from IL-10 ${ }^{\mathrm{tm} 1 / \mathrm{tm} 1}$ mice were prone to A1 phenotype}

IL-10 is a kind of anti-inflammatory factor and has a complex effect on innate immune activation status in the brain. To determine the role of IL-10 in astrocytes, B6.129S6-Il10 ${ }^{\mathrm{tm} 1 \mathrm{Flv}} / \mathrm{J}$ homozygotes (IL-10 ${ }^{\mathrm{tm} 1 / \mathrm{tm} 1}$ ) [22], which have reduced IL-10 expression, were used in this study. PCR data confirmed the genotype of IL-10 ${ }^{\mathrm{tm} 1 / \mathrm{tm} 1}$ mice (Fig. 2a). Astrocytes were isolated from neonatal WT and IL- $10^{\mathrm{tm} 1 / \mathrm{tm} 1}$ mice and then assessed for GFAP expression. Both immunofluorescence staining (Fig. 2b,

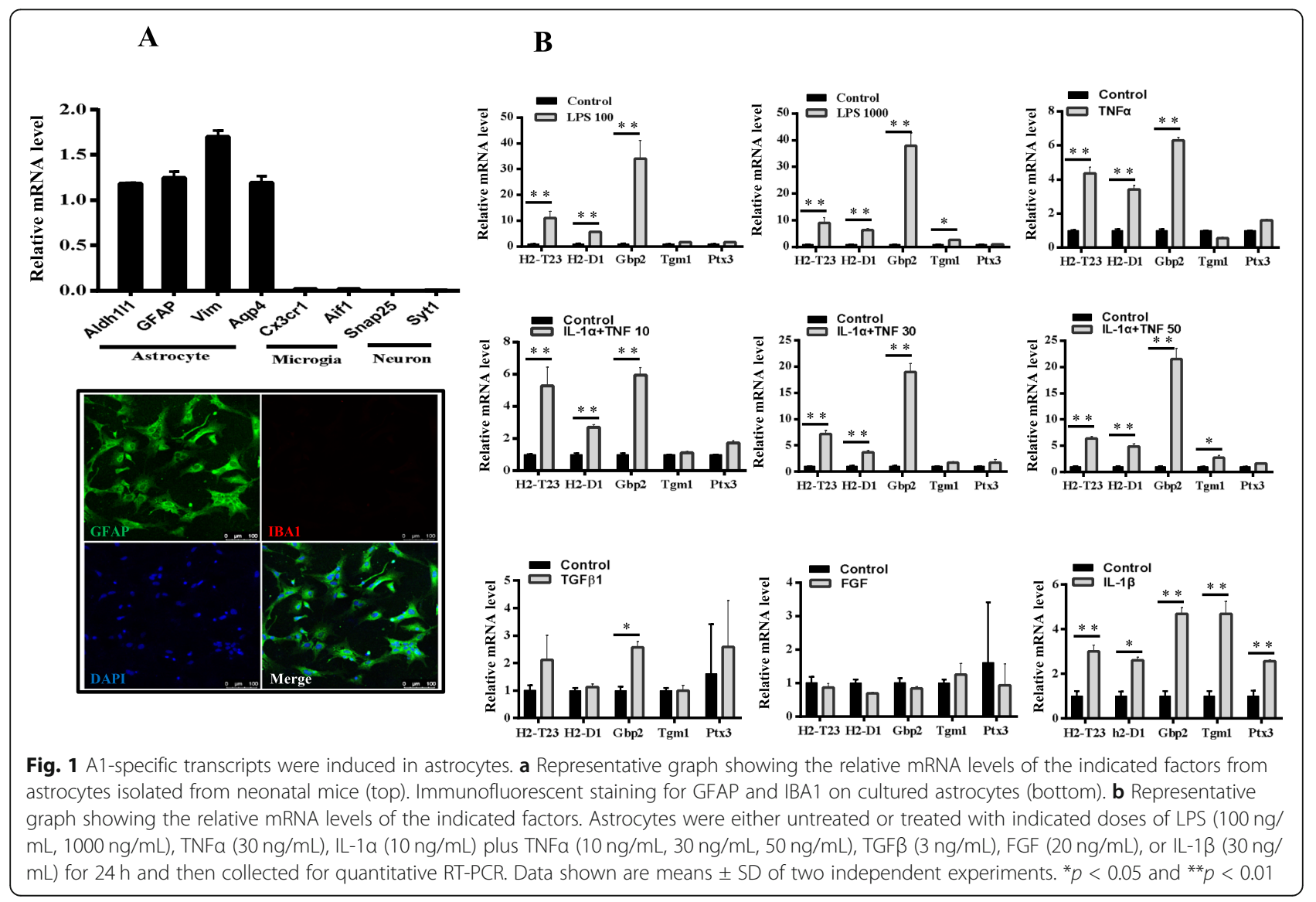




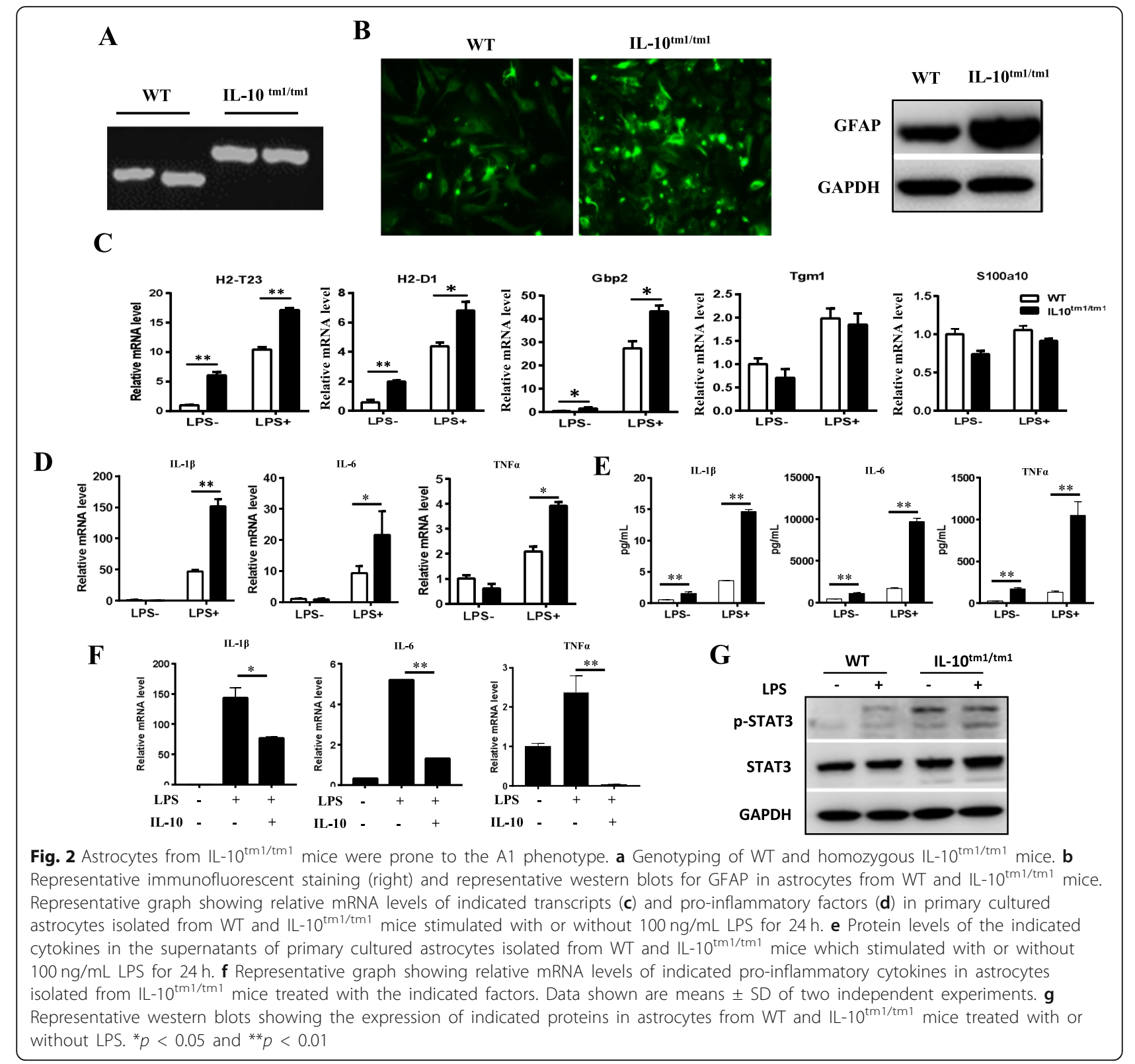

left) and immunoblot data (Fig. 2b, right) demonstrated a higher level of GFAP in IL-10 $0^{\mathrm{tm} 1 / \mathrm{tm} 1}$ astrocytes than in WT astrocytes. In addition, $\mathrm{IL}-10^{\mathrm{tm} 1 / \mathrm{tm} 1}$ astrocytes showed high expression of A1 transcripts H2-T23, H2D1, and Gbp2 under normal culture conditions. As expected, when stimulated with LPS, A1 transcripts H2T23, H2-D1, and Gbp2 were significantly induced. However, higher expression was detected in IL$10^{\mathrm{tm} 1 / \mathrm{tm} 1}$ astrocytes than in their WT counterparts, while A2 transcripts Tgm1 and S100a10 were comparable in WT and IL-10 ${ }^{\mathrm{tm} 1 / \mathrm{tm} 1}$ astrocytes (Fig. 2c). Next, we examined the expression of pro-inflammatory factors in $\mathrm{IL}-10^{\mathrm{tm} 1 / \mathrm{tm} 1}$ and WT astrocytes. Quantitative RT-PCR and ELISA analysis revealed that when stimulated with LPS, the expression of proinflammatory factors, including IL-1 $\beta$, IL-6, and TNF $\alpha$, was increased. Compared with their WT counterparts, $\mathrm{IL}-10^{\mathrm{tm} 1 / \mathrm{tm} 1}$ astrocytes demonstrated significantly enhanced expression of IL-1 $\beta$, IL-6, and TNF $\alpha$ (Fig. 2d, e). The LPS-induced expression of pro-inflammatory factors in $\mathrm{IL}-10^{\mathrm{tm} 1 / \mathrm{tm} 1}$ astrocytes was reduced by exogenous administration of IL-10 (Fig. 2f). In the CNS, STAT3 is a good candidate to be an activator of certain aspects of astrogliosis. As shown in Fig. 2g, coincident with higher expression of GFAP (Fig. 2b), phosphorylation of STAT3 was also enhanced in IL- $10^{\operatorname{tm} 1 / \mathrm{tm} 1}$ astrocytes, even stronger than that in WT astrocytes with LPS treatment. 
Astrocytes from IL-10 ${ }^{\mathrm{tm} 1 / \mathrm{tm} 1}$ mice showed attenuated normal astrocytic functions

Astrocytes are able to support neuron survival and induce the formation of functional synapses by secreting glypicans (Gpc4 and Gpc6), Sparcl1, and thrombospondins (Thbs1 and Thbs2). Next, we investigated the function of IL$10^{\mathrm{tm} 1 / \mathrm{tm} 1}$ astrocytes on neurons. WT and $\mathrm{IL}-10^{\mathrm{tm} 1 / \mathrm{tm} 1}$ astrocytes were stimulated with or without LPS for $24 \mathrm{~h}$, and then, their supernatant was collected. Purified neurons cultured from neonatal mice were treated with supernatant from WT and IL-10 $0^{\mathrm{tm} 1 / \mathrm{tm} 1}$ astrocytes. In contrast to support neuron survival, neurons treated with $\mathrm{IL}-10^{\mathrm{tm} 1 / \mathrm{tm} 1}$ astrocyte supernatant lost synaptic integrity (Fig. 3a). Correspondingly, LPS-treated astrocytes showed significantly decreased expression of Gpc4, Gpc6, and Thbs2 mRNA (Fig. 3b). Without LPS stimulation, IL- $10^{\mathrm{tm} 1 / \mathrm{tm} 1}$ astrocytes demonstrated lower expression of Gpc4, Gpc6, and Thbs2 than WT astrocytes but were comparable to WT astrocytes under LPS treatment. After treatment with LPS, IL- $10^{\mathrm{tm} 1 / \mathrm{tm} 1}$ astrocytes showed even lower expression of Gpc4, Gpc6, Thbs1, and Thbs2 (Fig. 3b). To examine the phagocytic ability of WT and IL-10 ${ }^{\mathrm{tm} 1 / \mathrm{tm} 1}$ astrocytes, engulfment of $A \beta 42$ was measured. Astrocytes can robustly phagocytose $A \beta 42$, but upon LPS stimulation, the ability significantly decreased (Fig. 3c). Compared with WT astrocytes, IL$10^{\mathrm{tm} 1 / \mathrm{tm} 1}$ astrocytes engulfed less $\mathrm{A} \beta 42$ whether or not they were treated with LPS. The phagocytic capacity corresponds with the expression of astrocyte-specific phagocytic receptors and bridging molecules. Decreased Mertk and Axl mRNA expression was found in $\mathrm{IL}-10^{\mathrm{tm} 1 / \mathrm{tm} 1}$ astrocytes, especially after LPS stimulation (Fig. 3d).

\section{IL-10 ${ }^{\mathrm{tm} 1 / \mathrm{tm} 1}$ mice showed higher expression of A1-specific} transcripts, enhanced neuroinflammation, and more neuron death

To examine whether lower IL-10 expression will affect the A1 phenotype and neuroinflammation in vivo and then contribute to neural death, the hippocampus and

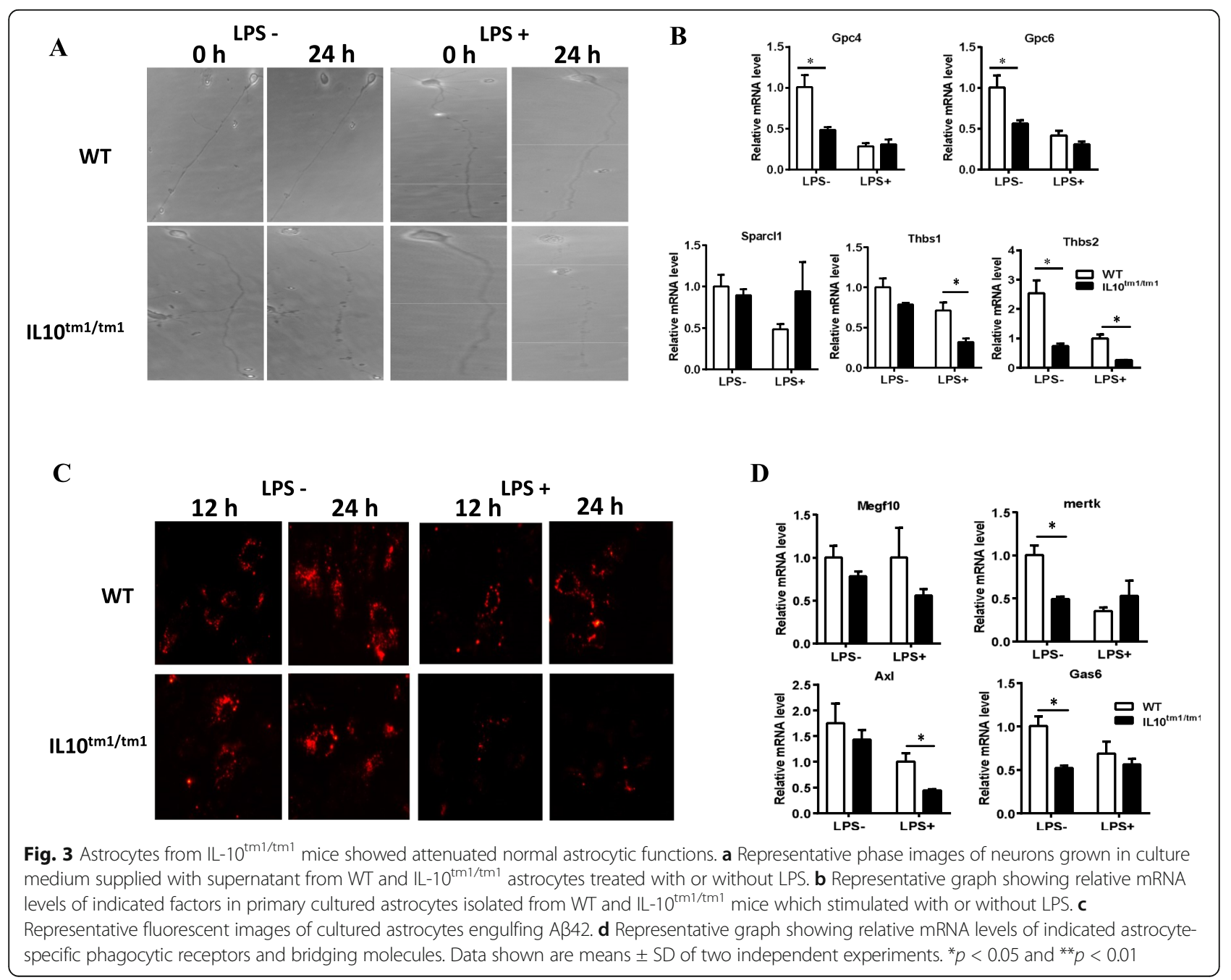


cortex were collected $24 \mathrm{~h}$ after intraperitoneal (i.p.) administration of LPS for quantitative RT-PCR analysis. As shown in Fig. 4a, b, A1-specific transcripts H2-T23, H2$\mathrm{D} 1$, and Gbp2 in the hippocampus and H2-D1 and Gbp2 in the cortex were significantly induced in IL$10^{\mathrm{tm} 1 / \mathrm{tm} 1}$ mice. Accordingly, quantitative RT-PCR (Fig. 4c) and ELISA (Fig. 4d) analysis revealed that the expression of pro-inflammatory factors IL-1 $\beta$, IL-6, and TNF $\alpha$ in the hippocampus was significantly higher than those from WT mice. In the cortex, higher levels of IL-6 and TNF $\alpha$ mRNA (Fig. 4e) and IL-6 protein (Fig. 4f) were found in $\mathrm{IL}-10^{\mathrm{tm} 1 / \mathrm{tm} 1}$ mice. In addition, immunofluorescent staining data demonstrated that more GFAP-positive cells were present in IL- $10^{\mathrm{tm} 1 / \mathrm{tm} 1}$ mice than in WT mice, which indicated the activation of astrocytes (Fig. 4g). A1 astrocytes and neuroinflammation will lead to neuron death. MAP2 immunostaining data revealed that compared with their WT counterparts, loss of MAP2-positive cells was observed in $\mathrm{IL}-10^{\mathrm{tm} 1 / \mathrm{tm} 1}$ mice (Fig. 4h).

\section{$\mathrm{IL}-10^{\mathrm{tm} 1 / \mathrm{tm} 1}$ mice exhibited depressive-like behavior and learning and memory deficits}

Studies have shown that A1 astrocytes are harmful and toxic to neurons and then affect the behaviors [5]. We then assessed potential depressive-like behaviors in IL$10^{\mathrm{tm} 1 / \mathrm{tm} 1}$ mice using the forced swim test (FST), which is a reliable behavioral assay for detecting depressant potential. Surprisingly, compared with their WT counterparts, IL-10 ${ }^{\operatorname{tm} 1 / \mathrm{tm} 1}$ mice showed a significant increase in immobility duration during the FST (Fig. 5a). LPS treatment can induce A1 astrocytes. We investigated the effect of LPS on the behaviors of both WT and IL$10^{\mathrm{tm} 1 / \mathrm{tm} 1}$ mice. After i.p. injection with LPS, there were

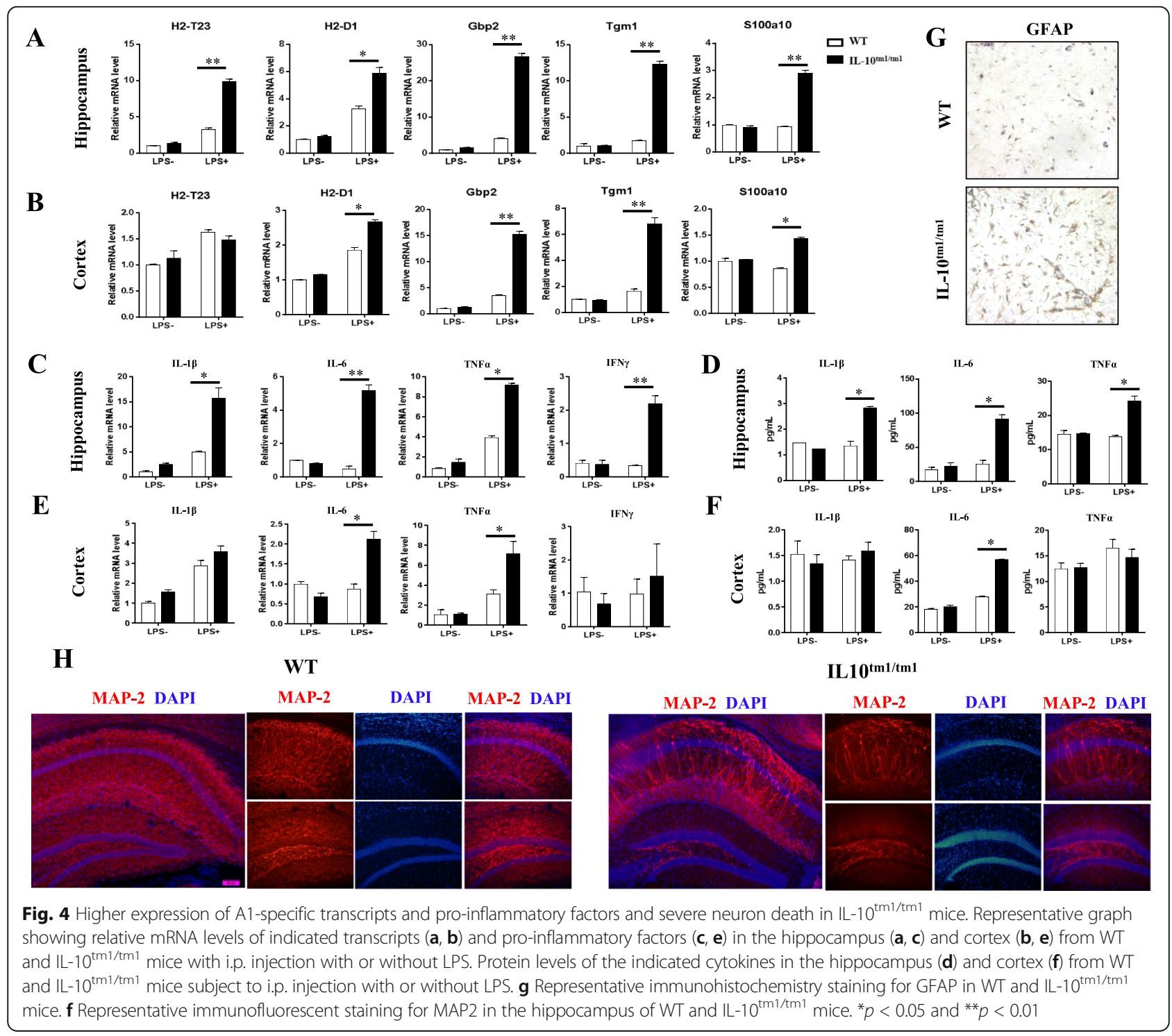


A

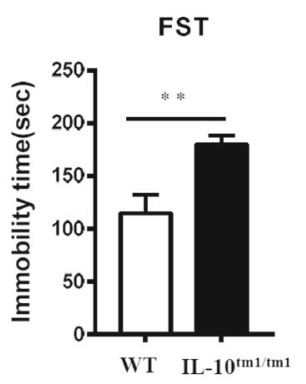

C

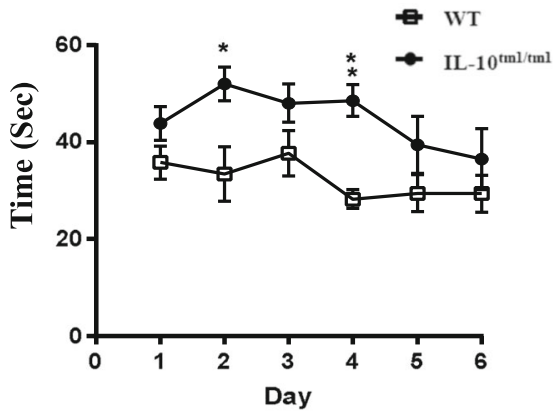

$\mathbf{E}$

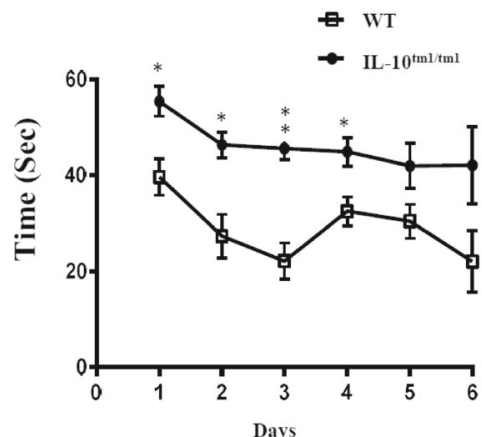

B

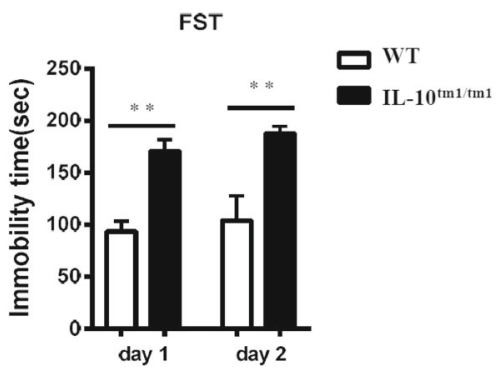

D
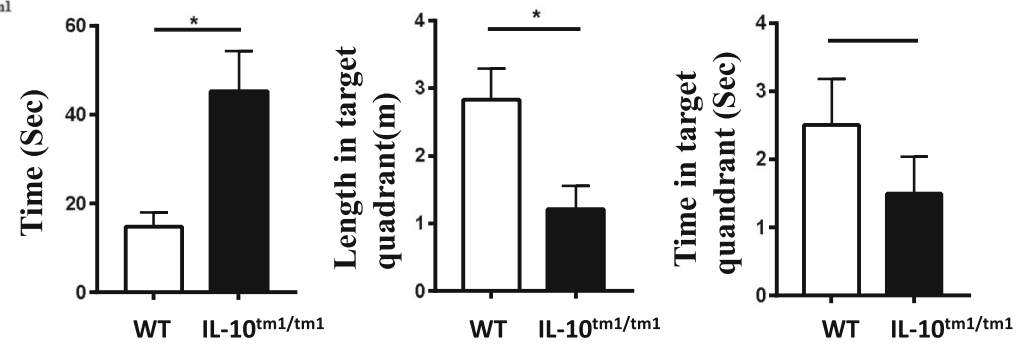

$\mathbf{F}$
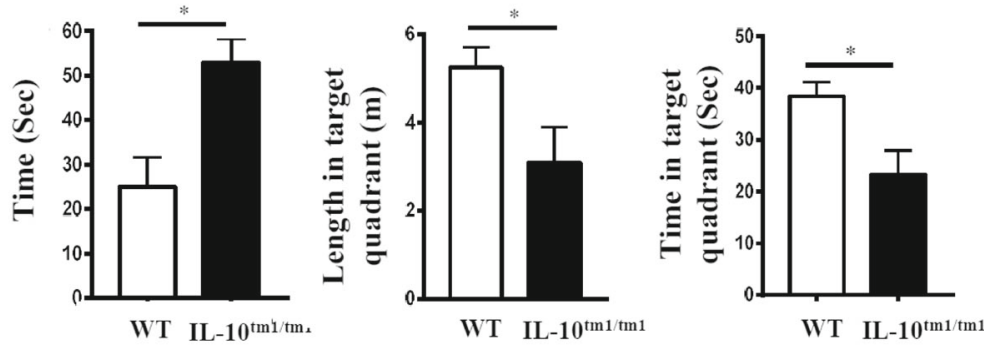

Fig. $5 \mathrm{LL}-10^{\mathrm{tm} 1 / \mathrm{tm} 1}$ mice exhibited depressive-like behavior and learning and memory deficits. a Representative graph showing the duration of immobility during the FST without LPS treatment. b Representative graph showing the duration of immobility during the FST recorded $24 \mathrm{~h}$ and $48 \mathrm{~h}$ following administration of LPS. MWMT performed over 6 days, including 5 days training and the 1-day test. The graphic shows the time (seconds) needed for each group of mice without (c) or with (e) LPS administration to localize a submerged platform in the swimming area. Representative graphs show escape latency (left), path length (middle), and time (right) spending in the target quadrant on the test day for each group of mice without (d) or with (f) LPS administration. ${ }^{*} p<0.05$ and ${ }^{* *} p<0.01$

more significant differences between the two groups, with increased time of immobility in $\mathrm{IL}-10^{\mathrm{tm} 1 / \mathrm{tm} 1}$ mice during FST, indicating severe depressive-like behavior (Fig. 5b). We then examined the spatial learning and memory of these mice through the Morris water maze test (MWMT) which reflects the functions of brain regions involved in learning and memory such as the hippocampus. Compared with WT mice, IL-10 ${ }^{\operatorname{tm} 1 / \mathrm{tm} 1}$ mice spent a significantly longer time exploring the quadrant that previously contained the platform and had a less number of crossings over the platform location (Fig. $5 \mathrm{c}, \mathrm{d}, \mathrm{e}, \mathrm{f}$ ). Together, these tests indicated that $\mathrm{IL}-10^{\operatorname{tm} 1 / \mathrm{tm} 1}$ mice exhibited depressive-like behavior and learning and memory deficits.

\section{IL-10 or FC treatment inhibited A1 astrocyte activation} and ameliorated LPS-induced behavior deficits

Previous studies have shown that FC can inhibit the activation of astrocytes and then ameliorates LPSinduced depressive-like behavior [24, 25]. To confirm the effect of A1 astrocyte activation and then on mouse behavior deficits, we analyzed the expression of A1 astrocyte-specific transcripts and proinflammatory cytokines in brain samples with IL-10 or FC pretreatment. IL- $10^{\mathrm{tm} 1 / \mathrm{tm} 1}$ mice were administered with IL-10 (20 ng, i.c.v), FC ( $1 \mathrm{nmol}$, i.c.v), or physiological saline for $6 \mathrm{~h}$ followed by a single i.p. injection of LPS $(0.83 \mathrm{mg} / \mathrm{kg})$. At $24 \mathrm{~h}$ after LPS injection, mice were submitted to tests for depressive-like behaviors and then were euthanized for collection of 
hippocampus and cortex samples. Quantitative RTPCR data showed that after IL-10 pretreatment, the expression of A1-specific transcripts Gbp2 in the hippocampus (Fig. 6a) and H2-T23, H2-D1, and Gbp2 in the cortex (Fig. 6b) were significantly decreased. Accordingly, attenuated expression of IL-1 $\beta$, IL-6, and TNF $\alpha$ was found both in the hippocampus (Fig. 6c) and cortex (Fig. 6d) from IL-10-pretreated IL$10^{\mathrm{tm} 1 / \mathrm{tm} 1}$ mice. Compared with saline-pretreated mice, IL-10 pretreatment significantly decreased the duration of immobility in the FST (Fig. 6e). Previous studies have shown that FC could inhibit astrocyte activation. Similarly, after FC pretreatment, the expression of A1-specific transcripts $\mathrm{H} 2-\mathrm{T} 23$ and H2-D1 in the hippocampus (Fig. 7a) and H2-T23 and H2-D1 in the cortex (Fig. 7b) were significantly decreased. Attenuated expression of IL-1 $\beta$, IL-6, and TNF $\alpha$ was also found both in the hippocampus (Fig. 7c), and lower IL-6 was observed in the cortex (Fig. 7d) from IL-10-pretreated IL-10 ${ }^{\mathrm{tm} 1 / \mathrm{tm} 1}$ mice. Compared with saline-pretreated mice, FC pretreatment also significantly decreased the duration of immobility in the FST (Fig. 7e).

\section{Discussion}

In this study, we found that astrocytes from IL-10 ${ }^{\mathrm{tm} 1 / \mathrm{tm} 1}$ mice, which have reduced IL-10 expression, were prone to A1 phenotype. After LPS injection, more A1 astrocytes were present in the brain tissues of $\mathrm{IL}-10^{\mathrm{tm} 1 / \mathrm{tm} 1}$ mice, which might contribute to their severe depressivelike behavior and learning and memory deficits. In addition, our data showed that IL-10 or FC pretreatment partially reduced the activation of $\mathrm{A} 1$ astrocytes and then ameliorated their behavior dysfunctions.

Astrocytes are abundant cells in the CNS and can be activated by many different types of molecules $[4,5,10$, 11, 26-29]. Here, we treated astrocytes isolated from neonatal mice with different cytokines and found that LPS, TNF $\alpha$, or IL- $1 \alpha$ plus TNF $\alpha$ can significantly induce the expression of A1 transcripts H2-T23, H2-D1, and Gbp2 but not A2 transcripts Tgm1 and Ptx3, which is similar to previous reports [8]. With LPS stimulation, astrocytes showed enhanced production of proinflammatory factors; decreased expression of Gpc4, Gpc6, Thbs1, and Thbs2; and reduced phagocytic ability. These indicated that the astrocytes in response to LPS showed an

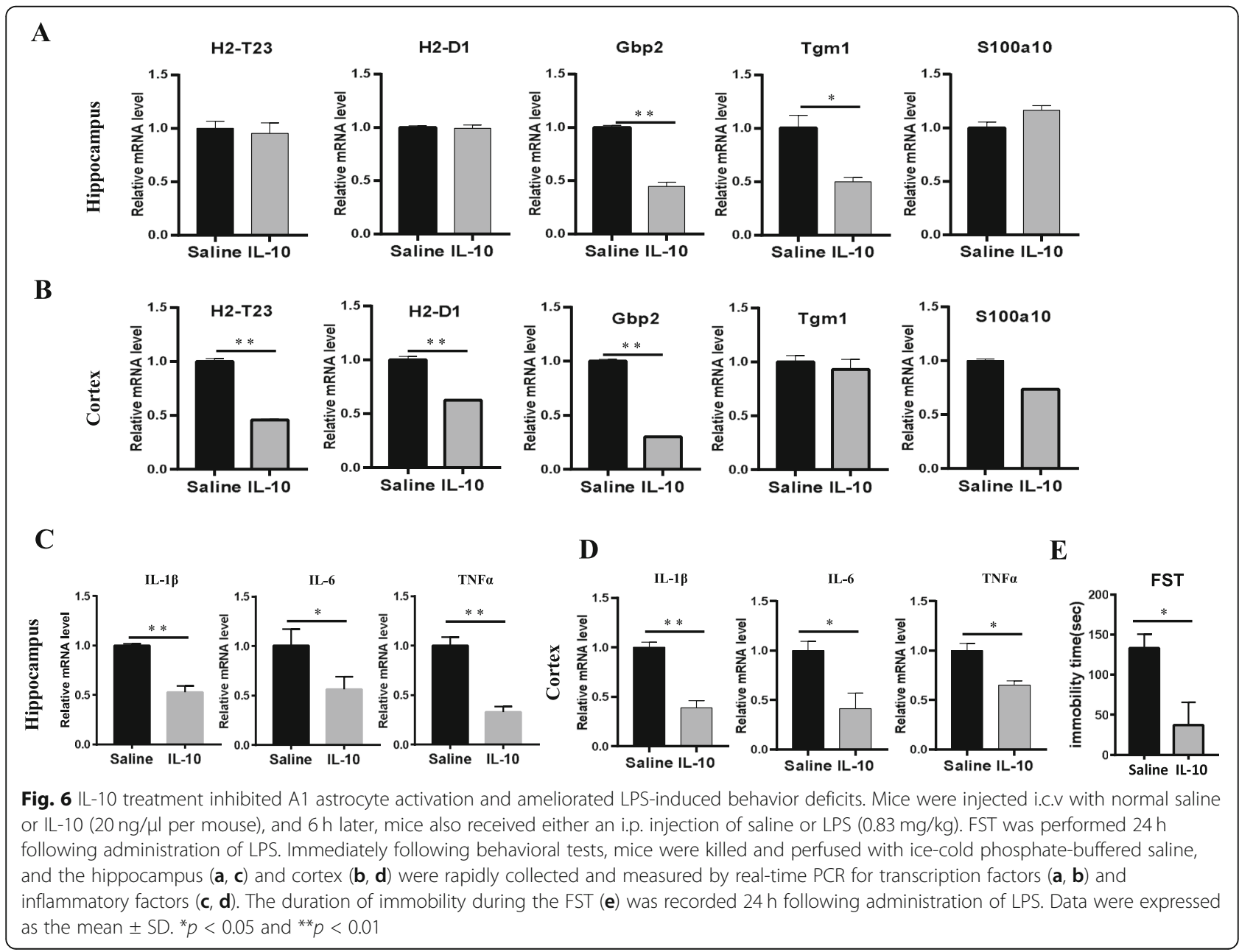




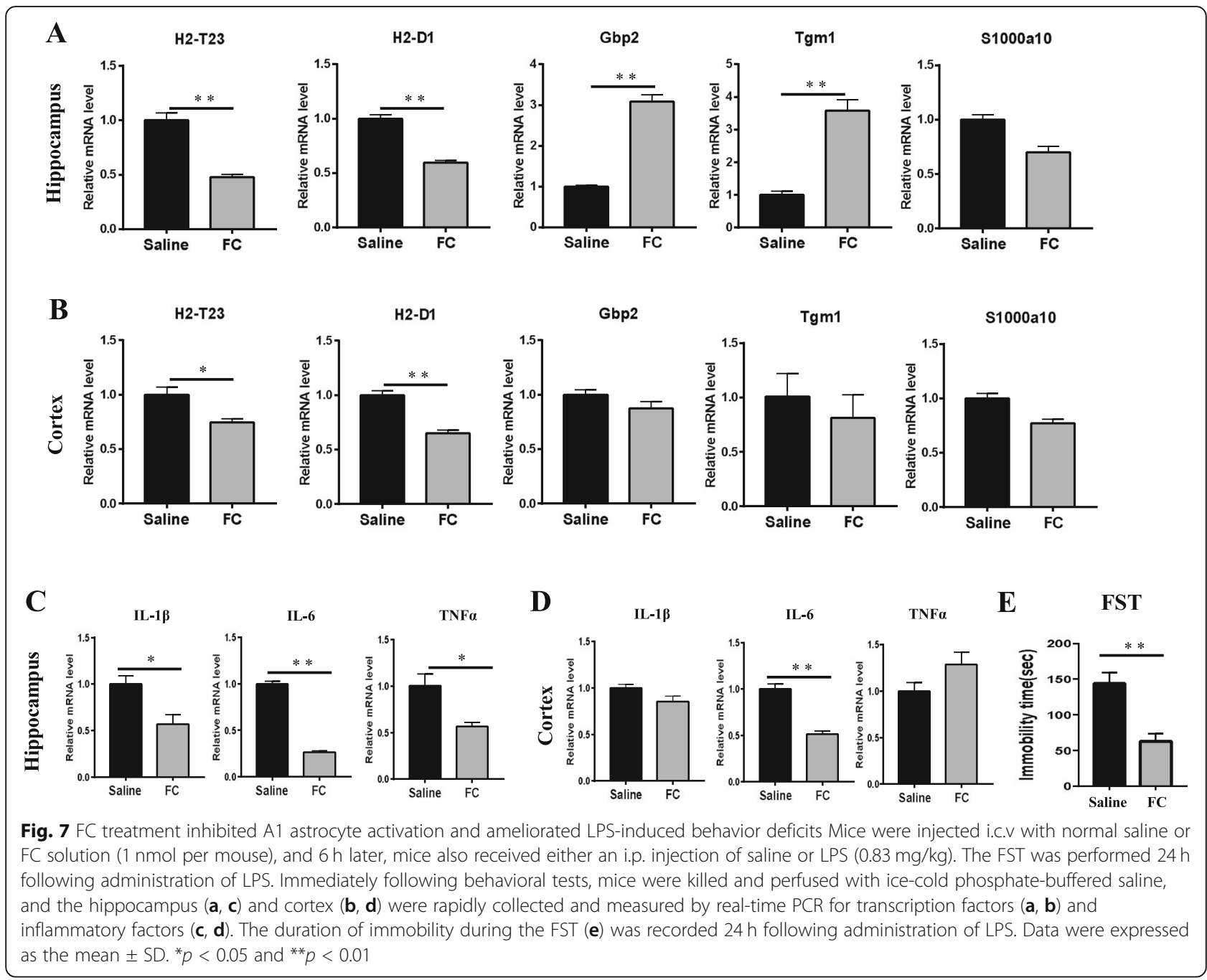

A1 characteristic $[8,9,24,30]$. B6.129S6-Il10 ${ }^{\mathrm{tm} 1 \mathrm{Flv}} / \mathrm{J}$ mice are generally used to detect and monitor cells committed to IL-10 production [22]. The homozygotes have reduced IL-10 expression. We found that astrocytes from B6.129S6-Il10 ${ }^{\mathrm{tm} 1 \mathrm{Flv}} / \mathrm{J}$ homozygotes (IL-10 ${ }^{\mathrm{tm} 1 / \mathrm{tm} 1}$ ) were prone to A1 astrocytes. Under normal culture condition, IL- $10^{\mathrm{tm} 1 / \mathrm{tm} 1}$ astrocytes demonstrated high expression of A1 transcripts. After treatment with LPS, both WT and IL-10 $0^{\mathrm{tm} 1 / \mathrm{tm} 1}$ astrocytes exhibited increased expression of A1 transcripts and proinflammatory factors, but the levels in IL-10 ${ }^{\mathrm{tm} 1 / \mathrm{tm} 1}$ astrocytes were much higher. In addition, compared to their WT counterparts, IL-10 ${ }^{\mathrm{tm} 1 / \mathrm{tm} 1}$ astrocytes were more toxic to neurons and less phagocytic, showing the characteristics of A1 phenotypes. What is more, compared with their counterparts, higher expression of A1 transcripts and proinflammatory factors was found in the hippocampus and cortex from IL-10 ${ }^{\mathrm{tm} 1 / \mathrm{tm} 1}$ mice. Liddelow et al. [8] have reported that reactive A1 astrocyte formation is a fundamental pathological response of many neurodegenerative diseases. A1 reactive astrocytes accumulated in aging brain and contribute to cognitive decline and a loss of memory. In addition to more severe depression-like behavior, IL$10^{\mathrm{tm} 1 / \mathrm{tm} 1}$ mice also exhibited more severe cognitive and memory deficits after being injected with the neuroinflammation inducer LPS. The behavior tests further confirmed the presence of $\mathrm{A} 1$ active astrocytes which secreted inflammatory factors IL- $1 \beta$, IL- 6, IFN $\gamma$, and TNF $\alpha$ and some unknown toxic factors inducing neurodegeneration, though we could not exclude the potential contribution of microglia cells in the brain.

Immune dysfunction is commonly associated with several neurological and mental disorders [31-36]. The innate immune system plays a central role in the CNS inflammation that drives neurological disability in progressive forms of multiple sclerosis, for which there are no effective treatments. It has been reported that cytokine imbalance is involved in the progression of many CNS diseases such as neuropsychiatric disorders and neurodegenerative disorders [13, 34, 37]. Enhanced IL- 
$1 \beta$ production in astrocytes is associated with the pathogenesis of major depressive disorder [38]. IFN- $\gamma$ deficient mice exhibited aberrant hyper-connectivity in frontal-cortical/insular regions though did not show anxiety or motor deficits [39]. SCID mice lacked social preference for a mouse over an object, but SCID mice did not show anxiety. IL-4-deficient mice did not demonstrate social deficits; in fact, they spent more time investigating a novel mouse than a novel object compared with WT mice. Further, IL-6 knockout mice show no difference in depressive-like behavior as measured by tail suspension or forced swim tests [40]. Altered IL-6 expression alone cannot account for depressive symptom, but clinical and animal studies showed that increased IL-6 and decreased IL10 will lead to depression [31, 41, 42]. IL-10-deficient female mice but not male mice displayed increased depressive-like behavior [43]. However, in our study, we found $\mathrm{IL}-10^{\mathrm{tm} 1 / \mathrm{tm} 1}$ male mice with IL-10 low expression exhibited increased immobility time in the forced swim test and defective learning and memory behaviors in the Morris water maze test. The activation of A1 astrocytes and the high expression of inflammatory factors IL-1 $\beta$, IL-6, IFN $\gamma$, and TNF $\alpha$ were mostly associated with the behavior deficits. Similar to some reports, mice pretreated with IL-10 presented a decreased depressive-like behavior $[41,43]$.

IL-10 is a cytokine classically linked with antiinflammatory and protective functions in the CNS in different neurodegenerative and neuroinflammatory conditions [15]. In the CNS, IL-10 is mainly produced by astrocytes and microglia and it is upregulated after various insults, such as experimental autoimmune encephalomyelitis, middle cerebral artery occlusion, excitotoxicity, and traumatic brain injury. Astrocytetargeted production of IL-10 impacted the microglial response and lymphocyte recruitment and culminated in a beneficial effect on neuronal survival [16]. However, elevated IL-10 is not always beneficial. In some AD patient brains, IL-10 signaling pathway was abnormally increased. In APP/PS1 mice, IL-10 deficiency was shown to partially rescue synaptic toxicity and mitigate behavioral impairment [20]. IL-10 overexpression in the brains of APP transgenic mice resulted in increased $A \beta$ accumulation and impaired memory [21]. The complex effect of IL-10 on innate immune activation status in the brain, proteostasis, and neurodegenerative diseases indicates controlling the right dose of IL-10 in the right condition is critical for brain development and function.

\section{Conclusion}

In conclusion, our data demonstrate that IL-10, an antiinflammatory cytokine, is an important molecule in the modulation of A1 astrocyte activation, depressive-like behavior, and learning and memory dysfunction. Furthermore, our data indicate that reducing the activation of A1 astrocytes may be an attractive therapy for some neuropsychiatric disorders or cognitive and memory deficits.

\begin{abstract}
Abbreviations
GFAP: Glial fibrillary acidic protein; LPS: Lipopolysaccharide; CNS: Central nervous system; NSCs: Neural stem cells; AD: Alzheimer's disease:

FC: Fluorocitrate; DAPI: 4',6-Diamidino-2-phenylindole; CSF: Cerebrospinal fluid; IBA1: Ionized calcium-binding adaptor molecule 1; FST: Forced swim test; MWMT: Morris water maze test
\end{abstract}

\section{Acknowledgements}

We thank Prof. Jianli Wang at Zhejiang University for providing B6.129S6$\| 10^{\text {tm1Flv/s }}$ mice.

\section{Authors' contributions}

XXJ conceived and designed the experiments. HYZ, YNQ, YW, YDH, XHH, TW, and $C M Z$ performed the experiments. $H Y Z, Y W, Y N Q, X H H, Y D H, S W L, C W$, and $X X J$ analyzed the experimental data. XXJ and YNQ wrote the manuscript. All authors read and approved the final manuscript.

\section{Funding}

This study was supported by grants from the National Natural Science Foundation of China (no. 81771998 to XXJ, no. 31971285 to XXJ, no. 31830030 to (W), Beijing Natural Science Foundation (no. 7202149 to XXJ), and National Key Research and Development Program of China (no. 2016YFE0204400 to YW, no. 2016YFC1101303 to CW).

\section{Availability of data and materials}

The datasets used and/or analyzed during the current study are available from the corresponding author upon reasonable request.

\section{Ethics approval and consent to participate}

All experimental animal protocols for this study are in accordance with the national guidelines for the use of animals in scientific research. Additional approval was granted by the Animal Care and Use Committee of the Academy of Military Medical Sciences.

Consent for publication

Not applicable.

\section{Competing interests}

The authors declare that they have no competing interests.

\section{Author details}

'Department of Neural Engineering and Biological Interdisciplinary Studies, Institute of Military Cognition and Brain Sciences, Academy of Military Medical Sciences, 27 Taiping Road, Haidian District, Beijing 100850, China ${ }^{2}$ Anhui Medical University, Hefei 230032, Anhui, China. ${ }^{3}$ Department of Neurology, Beijing Chaoyang Hospital, Capital Medical University, Beijing 100020, China. ${ }^{4}$ College of Agroforestry Engineering and Planning, Tongren University, Tongren 554300, Guizhou, China. ${ }^{5}$ Department of Geriatrics, Peking University Shenzhen Hospital, Shenzhen 518036, Guangzhou, China.

Received: 2 January 2020 Accepted: 10 June 2020

Published online: 01 July 2020

\section{References}

1. Khakh BS, Sofroniew MV. Diversity of astrocyte functions and phenotypes in neural circuits. Nat Neurosci. 2015;18(7):942-52.

2. Allen NJ, Eroglu C. Cell biology of astrocyte-synapse interactions. Neuron. 2017;96(3):697-708.

3. Bosworth AP, Allen NJ. The diverse actions of astrocytes during synaptic development. Curr Opin Neurobiol. 2017;47:38-43.

4. Anderson MA, Ao Y, Sofroniew MV. Heterogeneity of reactive astrocytes. Neurosci Lett. 2014;565:23-9. 
5. Liddelow SA, Barres BA. Reactive astrocytes: production, function, and therapeutic potential. Immunity. 2017;46(6):957-67.

6. Pavlou MAS, et al. Transcriptional and epigenetic mechanisms underlying astrocyte identity. Prog Neurobiol. 2019;174:36-52.

7. Kodack DP, et al. Emerging strategies for treating brain metastases from breast cancer. Cancer Cell. 2015;27(2):163-75.

8. Liddelow SA, et al. Neurotoxic reactive astrocytes are induced by activated microglia. Nature. 2017;541(7638):481-7.

9. Yun SP, et al. Block of A1 astrocyte conversion by microglia is neuroprotective in models of Parkinson's disease. Nat Med. 2018;24(7):931-8.

10. Hinkle JT, Dawson VL, Dawson TM. The A1 astrocyte paradigm: new avenues for pharmacological intervention in neurodegeneration. Mov Disord. 2019;34(7):959-69.

11. Okada $\mathrm{S}$, et al. Astrocyte reactivity and astrogliosis after spinal cord injury. Neurosci Res. 2018;126:39-43.

12. Perez-Nievas BG, Serrano-Pozo A. Deciphering the astrocyte reaction in Alzheimer's disease. Front Aging Neurosci. 2018;10:114.

13. Banchereau J, Pascual V, O'Garra A. From IL-2 to IL-37: the expanding spectrum of anti-inflammatory cytokines. Nat Immunol. 2012;13(10):925-31.

14. Recasens $\mathrm{M}$, et al. Astrocyte-targeted IL-10 production decreases proliferation and induces a downregulation of activated microglia/ macrophages after PPT. Glia. 2019;67(4):741-58.

15. Almolda B, et al. Alterations in microglial phenotype and hippocampal neuronal function in transgenic mice with astrocyte-targeted production of interleukin-10. Brain Behav Immun. 2015;45:80-97.

16. Villacampa N, et al. Astrocyte-targeted production of IL-10 induces changes in microglial reactivity and reduces motor neuron death after facial nerve axotomy. Glia. 2015;63(7):1166-84

17. Yang J, et al. Adult neural stem cells expressing IL-10 confer potent immunomodulation and remyelination in experimental autoimmune encephalitis. J Clin Invest. 2009;1 19(12):3678-91.

18. Perez-Asensio FJ, et al. Interleukin-10 regulates progenitor differentiation and modulates neurogenesis in adult brain. J Cell Sci. 2013;126(Pt 18): 4208-19.

19. Pereira $L$, et al. IL-10 regulates adult neurogenesis by modulating ERK and STAT3 activity. Front Cell Neurosci. 2015:9:57.

20. Guillot-Sestier MV, et al. II10 deficiency rebalances innate immunity to mitigate Alzheimer-like pathology. Neuron. 2015;85(3):534-48.

21. Chakrabarty P, et al. IL-10 alters immunoproteostasis in APP mice, increasing plaque burden and worsening cognitive behavior. Neuron. 2015;85(3):519-33.

22. Kamanaka $\mathrm{M}$, et al. Expression of interleukin-10 in intestinal lymphocytes detected by an interleukin-10 reporter knockin tiger mouse. Immunity. 2006 25(6):941-52.

23. O'Connor JC, et al. Lipopolysaccharide-induced depressive-like behavior is mediated by indoleamine 2,3-dioxygenase activation in mice. Mol Psychiatry. 2009;14(5):511-22.

24. Zhang $X$, et al. Inhibition of reactive astrocytes with fluorocitrate ameliorates learning and memory impairment through upregulating CRTC1 and synaptophysin in ischemic stroke rats. Cell Mol Neurobiol. 2019;39(8):1151-63

25. Wang $Y$, et al. Inhibition of activated astrocyte ameliorates lipopolysaccharideinduced depressive-like behaviors. J Affect Disord. 2019;242:52-9.

26. Sofroniew MV, Vinters HV. Astrocytes: biology and pathology. Acta Neuropathol. 2010;119(1):7-35.

27. Faulkner JR, et al. Reactive astrocytes protect tissue and preserve function after spinal cord injury. J Neurosci. 2004;24(9):2143-55.

28. Buffo A, Rolando C, Ceruti S. Astrocytes in the damaged brain: molecular and cellular insights into their reactive response and healing potential. Biochem Pharmacol. 2010;79(2):77-89.

29. Pekny M, Pekna M. Astrocyte reactivity and reactive astrogliosis: costs and benefits. Physiol Rev. 2014;94(4):1077-98.

30. Clarkea $L E$, et al. Normal aging induces A1-like astrocyte reactivity. Proc Natl Acad Sci U S A. 2018;115:E1896-905.

31. Dantzer $R$, et al. Inflammation-associated depression: from serotonin to kynurenine. Psychoneuroendocrinology. 2011;36(3):426-36.

32. Shao $W$, et al. Suppression of neuroinflammation by astrocytic dopamine D2 receptors via alphaB-crystallin. Nature. 2013;494(7435):90-4.

33. Wood SK, et al. Inflammatory factors mediate vulnerability to a social stressinduced depressive-like phenotype in passive coping rats. Biol Psychiatry. 2015;78(1):38-48
34. Bellaver B, et al. Systemic inflammation as a driver of brain injury: the astrocyte as an emerging player. Mol Neurobiol. 2018;55(3):2685-95.

35. Santos $\mathrm{CL}$, et al. Leptin stimulates the release of pro-inflammatory cytokines in hypothalamic astrocyte cultures from adult and aged rats. Metab Brain Dis. 2018;33(6):2059-63.

36. Grimaldi A, et al. Neuroinflammatory processes, A1 astrocyte activation and protein aggregation in the retina of Alzheimer's disease patients, possible biomarkers for early diagnosis. Front Neurosci. 2019;13:925.

37. Dossi E, Vasile F, Rouach N. Human astrocytes in the diseased brain. Brain Res Bull. 2018;136:139-56.

38. Leng $L$, et al. Menin deficiency leads to depressive-like behaviors in mice by modulating astrocyte-mediated neuroinflammation. Neuron. 2018;100(3): 551-63 e7.

39. Filiano AJ, et al. Unexpected role of interferon-gamma in regulating neuronal connectivity and social behaviour. Nature. 2016;535(7612):425-9.

40. Swiergiel AH, Dunn AJ. Feeding, exploratory, anxiety- and depressionrelated behaviors are not altered in interleukin-6-deficient male mice. Behav Brain Res. 2006;171(1):94-108.

41. Voorhees $\mathrm{JL}$, et al. Prolonged restraint stress increases IL-6, reduces IL-10, and causes persistent depressive-like behavior that is reversed by recombinant IL-10. PLoS One. 2013;8(3):e58488.

42. Labaka A, et al. Reduced hippocampal IL-10 expression, altered monoaminergic activity and anxiety and depressive-like behavior in female mice subjected to chronic social instability stress. Behav Brain Res. 2017;335: $8-18$.

43. Mesquita AR, et al. IL-10 modulates depressive-like behavior. J Psychiatr Res. 2008:43(2):89-97.

\section{Publisher's Note}

Springer Nature remains neutral with regard to jurisdictional claims in published maps and institutional affiliations.
Ready to submit your research? Choose BMC and benefit from:

- fast, convenient online submission

- thorough peer review by experienced researchers in your field

- rapid publication on acceptance

- support for research data, including large and complex data types

- gold Open Access which fosters wider collaboration and increased citations

- maximum visibility for your research: over $100 \mathrm{M}$ website views per year

At BMC, research is always in progress.

Learn more biomedcentral.com/submissions 